\title{
Other Food Preparation and Serving Related Workers
}

National Cancer Institute

\section{Source}

National Cancer Institute. Other Food Preparation and Serving Related Workers. NCI

Thesaurus. Code C122543.

Food preparation and serving related workers not specifically identified elsewhere. 